\title{
Exchange interaction in binuclear complexes with rare-earth and copper ions: A many-body model study
}

\author{
Indranil Rudra, C. Raghu, and S. Ramasesha \\ Solid State and Structural Chemistry Unit, Indian Institute of Science, Bangalore 560 012, India
}

(Received 12 December 2001; published 28 May 2002)

\begin{abstract}
We have used a many-body model Hamiltonian to study the nature of the magnetic ground state of heterobinuclear complexes involving rare-earth and copper ions. We have taken into account all diagonal repulsions involving the rare-earth $4 f$ and $5 d$ orbitals and the copper $3 d$ orbital. In addition, we have included direct exchange interaction, crystal field splitting of the rare-earth atomic levels and spin-orbit interaction in the $4 f$ orbitals. We have identified the interorbital $4 f$ repulsion $U_{f f}$ and crystal field parameter $\Delta_{f}$ as the key parameters involved in controlling the type of exchange interaction between the rare earth $4 f$ and copper $3 d$ spins. We have explored the nature of the ground state in the parameter space of $U_{f f}, \Delta_{f}$, spin-orbit interaction strength $\lambda$, and the $4 f$ filling $n_{f}$. We find that these systems show low-spin or high-spin ground state depending on the filling of the $4 f$ levels of the rare-earth ion and ground state spin is critically dependent on $U_{f f}$ and $\Delta_{f}$. In case of half filling [Gd(III)] we find a reentrant low-spin state as $U_{f f}$ is increased, for small values of $\Delta_{f}$, which explains the recently reported apparent anomalous antiferromagnetic behavior of Gd(III)-radical complexes. By varying $U_{f f}$ we also observe a switch over in the ground state spin for other fillings. We have introduced a spin-orbit coupling scheme which goes beyond the $L-S$ or $j-j$ coupling scheme and we find that spin-orbit coupling does not significantly alter the basic picture.
\end{abstract}

DOI: 10.1103/PhysRevB.65.224411

PACS number(s): 75.50.Xx, 75.30.-m

\section{INTRODUCTION}

Electron spin pairing and the associated electron exchange between atoms is the cornerstone of chemical bond formation. Hence most of the molecules have closed shell ground state ${ }^{1}$ and the synthesis of high spin molecules as well as molecular magnets has been a challenge. The simplest idea to synthesize a high spin molecule is to start with a high spin carrier. In this regard lanthanides, and in particular, $\mathrm{Gd}^{3+}$ which has a $4 f^{7}$ configuration is an obvious choice. Mononuclear complexes of $\mathrm{Gd}^{3+}$ are already known. Systems with ground state spin higher than $\frac{7}{2}$ can be built up with multinuclear complexes involving $\mathrm{Gd}^{3+}$ and other rareearth or transition metal ions. Studies of such heteropolymetallic compounds have also been of great interest in modeling metaloenzymes and in understanding their magnetic properties. $^{2}$ Indeed, the interaction between two nonequivalent metal centers can lead to some unique features which are not encountered in complexes with the same kind of spin carriers.

In the literature, there have been several studies of heteropolymetallic molecular coordination complexes of lanthanides and $\mathrm{Cu}(\mathrm{II})$ or other ions because of their luminescence and interesting magnetic properties. ${ }^{3}$ Some of these complexes exhibit a high spin ground state corresponding to a ferromagnetic alignment of magnetic moments of the individual ions, a fact which is important for designing building blocks for molecular magnets. Gatteschi and co-workers ${ }^{4-6}$ found that in the case of $\mathrm{Gd}(\mathrm{III})-\mathrm{Cu}(\mathrm{II})$ binuclear and $\mathrm{Cu}(\mathrm{II})$ $\mathrm{Gd}(\mathrm{III})-\mathrm{Cu}(\mathrm{II})$ trinuclear complexes, the $\mathrm{Gd}(\mathrm{III})-\mathrm{Cu}(\mathrm{II})$ interaction is ferromagnetic in nature, independent of the structure of the complex or the ligand molecule. Subsequently, a systematic effort to synthesize and study binuclear systems between $\mathrm{Cu}(\mathrm{II})$ and other rare-earth ions was undertaken. ${ }^{7-9}$
These studies indicate a general trend for the ground state spin of the complex as we traverse the lanthanide series. The interaction between $\mathrm{Cu}(\mathrm{II})-\mathrm{Ln}$ (III) is ferromagnetic for $\mathrm{Gd}(\mathrm{III})$ and other rare-earth ions with greater than half-filled $4 f$ shell such as $\mathrm{Dy}(\mathrm{III})$ and $\operatorname{Er}(\mathrm{III})$. Below half filling, this interaction is antiferromagnetic and leads to low-spin ground state for systems such as $\mathrm{Cu}(\mathrm{II})-\mathrm{Ce}(\mathrm{III})$ and $\mathrm{Cu}(\mathrm{II})-\mathrm{Eu}(\mathrm{III}){ }^{7}$ The situation remains the same when the $\mathrm{Cu}$ (II) is replaced by organic ligands such as nitronyl nitroxide triazole which are essentially spin- $1 / 2$ carriers.

As a possible mechanism for the observed ferromagnetic alignment of the spin of the rare-earth ion with the spin of the copper ion, it was pointed out ${ }^{10}$ that the stabilization of the high spin state arises from the coupling between the ground-state configuration and the excited-state configuration arising out of the transfer of the electron from the singly occupied copper $3 d$ orbital to an empty $5 d$ orbital of the rare-earth ion. This mechanism is in the spirit of the superexchange mechanisms proposed by Goodenough ${ }^{13}$ in 1963. Similar mechanism is invoked recently by Tchougreeff ${ }^{14}$ to explain the ferromagnetic ordering in decamethylferrocenium tetracyanoethenide, by Kinoshita and co-workers ${ }^{15}$ to explain the intermolecular ferromagnetic coupling in paranitrophenyl nitronyl nitroxide and by Girerd and co-workers ${ }^{16}$ to interpret the magnetic properties of $\mu$-oxo $\mathrm{Mn}$ (II) compounds. Indeed, the interplay of direct exchange which favors parallel spin alignment on a given site (Hund's rule) and kinetic exchange which favours antiparallel alignment of spins on bonded pair of sites (by virtue of Pauli principle) is what determines the spin state of a system. ${ }^{17}$

Kahn and co-workers modeled the superexchange process using an extended Hückel model and obtained a value for the septet-nonet gap from the computed $J_{\mathrm{Gd}-\mathrm{Cu}}$ in fairly good agreement with the experimental result. But in this model 
strong electron-electron repulsions among the $4 f$ electrons arising out of the contraction of the lanthanide $4 f$ orbitals as well as between $4 f$ electrons and the $5 d$ electrons have not been taken into account. These electron repulsions, according to the recent reports seems to play an important role in explaining the photoemission spectra of rare-earth compounds. ${ }^{18}$ Another factor which was not accounted for in the above model is the splitting of the free ion $\mathrm{Ln}$ (III) ground state due to the ligand field effect, on the grounds that spinorbit coupling term is an order of magnitude larger than crystal field term. ${ }^{19}$ The need of a detailed microscopic model taking these factors into account has also been felt since some exceptions to ferromagnetic coupling in Gd(III) complexes were recently reported for $\mathrm{Cu}(\mathrm{II})$ complexes and nitronyl nitroxide radicals. ${ }^{20-22}$ To explain this unexpected antiferromagnetic coupling, suggestions were put forward that the observed coupling is actually the sum of two contributions, ${ }^{22}$ one from the direct overlap of the magnetic orbitals of the ligand with the $f$ orbitals, which leads to antiferromagnetism and the other from the overlap with the $s$ and $d$ orbitals, which results in ferromagnetism. But the rareearth $f$ orbitals are highly contracted to the core which prompted Kahn et al. ${ }^{10}$ to neglect the transfer between the $4 f$ orbitals of $\mathrm{Ln}(\mathrm{III})$ and $3 d$ orbitals of $\mathrm{Cu}(\mathrm{II})$.

In this paper we present our studies of a detailed microscopic model in which we have taken into account explicit electron-electron correlations, crystal field effects and spinorbit effects in the lanthanide ion. We have spanned the parameter space of the model and have obtained phase diagrams for different fillings of the $4 f$ orbitals to elucidate the role played by the different parameters in controlling the ground state spin. The paper is organized as follows. In Sec. II, we introduce the many-body model Hamiltonian. We also briefly describe the computational schemes employed in solving the model Hamiltonian. In Sec. III we discuss the results obtained by our computations on the model. Finally, we summarize our results in Sec. IV.

\section{MANY-BODY MODEL AND COMPUTATIONAL SCHEME}

The remarkable feature of these systems is that the parallel or antiparallel alignment of spins in the ground state is independent of the details of structure. This led Kahn et al. to propose a general mechanism by the virtue of which these systems attain either the high-spin or the low-spin state. The contraction of the $4 f$ orbitals towards the nucleus means that they are very weakly delocalized towards the $\mathrm{Cu} 3 d$ orbitals and thus, there is a vanishingly small overlap between the $\mathrm{Gd}$ $4 f$ and $\mathrm{Cu} 3 d$ orbitals. This also means that any interaction between the ground-state configuration and the charge transfer configuration corresponding to a $3 d-4 f$ transfer cannot stabilize the low-spin $S=3$ state. So, Kahn et al. neglected these $3 d-4 f$ interactions and considered charge transfer to the higher $5 d$ level, i.e., the $3 d$ electron being transfered to an empty $5 d$ orbital of the $\mathrm{Gd}(\mathrm{III})$ ion. This process results in the $S=4$ high-spin state being stabilized due to a strong Hund's coupling between the $4 f$ and $5 d$ levels. This process is illustrated in Fig. 1. The mechanism outlined by Kahn

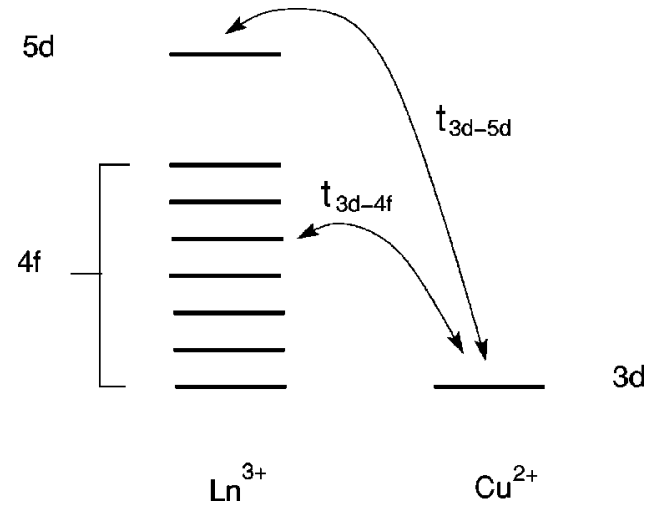

FIG. 1. A schematic for the mechanism which leads to the stabilization of the high-spin state in the system. $\mathrm{Ln}^{3+}$ is the rare-earth (lanthanide) ion. Transfers between the $\mathrm{Cu}^{2+} 3 d$ and the $\mathrm{Ln} 4 f$ and $5 d$ levels are indicated by the arrows. The $3 d \rightarrow 4 f$ transfer leads to a higher-energy virtual state than $3 d \rightarrow 5 d$ transfer.

et al. is, however, still in a noninteracting picture since it does not take into account explicit Coulomb repulsions among the electrons in different orbitals. Another factor missing in the model is the effect of the crystal-field interaction on the various orbitals involved. In order to take these effects into account and understand how they modify the ground-state spin in these systems, we have studied these systems by employing an interacting Hamiltonian. This Hamiltonian is an extension of the Pariser-Parr-Pople model $^{11}$ to more than one orbital per atom and includes direct exchange interaction terms between electrons in orbitals on the same site. This model has been greatly successful in describing ground state and low-lying excited states of various strongly correlated systems.

When we have more than one orbital per site, the ZDO (zero differential overlap) approximation is not valid and we should include electron repulsion integrals when two electrons are in different orbitals on the same atom. In this case, we will have several kinds of nonzero two electron integrals on the same site. The interaction part of the Hamiltonian in this modified ZDO approximation is given by

$$
\begin{aligned}
H_{\mathrm{int}}= & 1 / 2 \sum_{i, j} \sum_{\mu, \mu^{\prime}, \nu, \nu^{\prime}}\left[i_{\mu} i_{\mu^{\prime}} \mid j_{\nu} j_{\nu^{\prime}}\right]\left(\hat{E}_{i \mu, i \mu^{\prime}} \hat{E}_{j \nu, j \nu^{\prime}}\right. \\
& \left.-\delta_{i j} \delta_{\mu^{\prime} \nu} \hat{E}_{i \mu, j \nu^{\prime}}\right) .
\end{aligned}
$$

Here, $i, j$ are site indices and $\mu, \nu$ correspond to the orbital on a particular site. The two electron integrals $\left[i_{\mu} i_{\mu^{\prime}} \mid j_{\nu} j_{\nu^{\prime}}\right]$ contains contributions from various kinds of situations. The case $\mu=\mu^{\prime}=\nu=\nu^{\prime}$ and $i=j$ corresponds to the electronrepulsion integral for the electrons in the same orbital of a single site. The most general case for the repulsion integral on the same site is realized when there are at least four orbitals per site and $\mu \neq \mu^{\prime} \neq \nu \neq \nu^{\prime}$ and $i=j$. For the $f$ orbitals on the same site, we have assumed that (i) the intraorbital repulsion integral $\left[f_{\mu} f_{\mu} \mid f_{\mu} f_{\mu}\right]$ is the same for all the seven $f$ orbitals, independent of $\mu$, and given by $U_{f}$, (ii) the inter- $f$ orbital repulsion integral $\left[f_{\mu} f_{\mu} \mid f_{\nu} f_{\nu}\right]$ is also independent of the particular pair of $f$ orbitals $\mu, \nu$ and given by $U_{f f}$, and 
(iii) that there is also a single direct exchange integral $J_{f f}$ between two $f$ orbitals corresponding to the integral $\left[f_{\mu} f_{\nu} \mid f_{\mu} f_{\nu}\right]$. We have further assumed that all other electron repulsion integrals involving the $f$ orbitals are zero. The direct exchange integral although smaller than the electron repulsion integrals in (i) and (ii) by about an order of magnitude, is crucial to the study of magnetic gaps. Indeed, the small value of the exchange integral is also reflected in the small value of the magnetic gaps. For our rare-earth system, in most cases, we have considered all the seven $4 f$ orbitals and one $5 d$ orbitals for the rare-earth metal ion and a single
$3 d$ orbital for the $\mathrm{Cu}$ ion. Inclusion of $5 \mathrm{~d}$ orbital introduces corresponding electron repulsion parameters $U_{5 d}, U_{f d}$, and $J_{f d}$. Electron repulsion integrals of the form $\left[i_{\mu} i_{\mu} \mid j_{\nu} j_{\nu}\right]$ which in our case corresponds to repulsion integral $V_{f d}$ between an electron in the $4 f$ orbital of the rare-earth and an electron in the $3 d$ orbital of the $\mathrm{Cu}$ ion is parametrized using Ohno interpolation scheme. ${ }^{12}$ All other intersite electron repulsion integrals are neglected.

Using the above parameters, the model Hamiltonian is written as

$$
\begin{aligned}
\hat{H}= & \sum_{f=1}^{7} \Delta_{f} f \hat{n}_{f}+\Delta_{3 d} \hat{n}_{3 d}+\Delta_{5 d} \hat{n}_{5 d^{\prime}}+t_{3 d-4 f} \sum_{f=1}^{7}\left(\hat{E}_{f, 3 d}+\text { H.c. }\right)+t_{3 d-5 d}\left(\hat{E}_{3 d, 5 d}+\text { H.c. }\right)+\frac{U_{f}}{2} \sum_{f=1}^{7} \hat{n}_{f}\left(\hat{n}_{f}-1\right)+\frac{U_{3 d}}{2} \hat{n}_{3 d}\left(\hat{n}_{3 d}-1\right) \\
& +\frac{U_{5 d}}{2} \hat{n}_{5 d}\left(\hat{n}_{5 d}-1\right)+U_{f f} \sum_{f>f^{\prime}=1}^{7} \hat{n}_{f} \hat{n}_{f^{\prime}}+U_{f d} \sum_{f=1}^{7} \hat{n}_{f} \hat{n}_{5 d}+V_{3 d-4 f} \sum_{f=1}^{7} \hat{n}_{f} \hat{n}_{3 d}+V_{3 d-5 d} \hat{n}_{3 d} \hat{n}_{5 d}+J_{f f} \\
& \times \sum_{f>f^{\prime}=1}^{7}\left[\left(\hat{E}_{f, f^{\prime}} \hat{E}_{f, f^{\prime}}+\hat{E}_{f, f^{\prime}} \hat{E}_{f^{\prime}, f^{\prime}}+\text { H. c. }\right)-\hat{n}_{f}-\hat{n}_{f^{\prime}}\right]+J_{f d} \sum_{f=1}^{7}\left[\left(\hat{E}_{f, 5 d} \hat{E}_{f, 5 d}+\hat{E}_{5 d, f} \hat{E}_{f, 5 d}+\text { H. c. }\right)-\hat{n}_{f}-\hat{n}_{5 d}\right]
\end{aligned}
$$

where $\hat{E}_{i j}=\Sigma_{\sigma} \hat{a}_{i, \sigma}^{\dagger} \hat{a}_{j, \sigma}$ defines the electron hop operator $\hat{E}_{i j}$ which hops an electron of either spin from orbital $j$ to orbital $i, \hat{a}_{i, \sigma}^{\dagger}\left(\hat{a}_{i, \sigma}\right)$ creates (annihilates) an electron with spin $\sigma$ in the $i$ th orbital. The first two lines in Eq. (2) corresponds to the noninteracting part of the Hamiltonian. The first line corresponds to the site energy contribution. Here, we assume that the degeneracy of the $4 f$ levels are lifted uniformly by the nonsymmetric crystalline field, leading to equally spaced levels with an energy level spacing $\Delta_{f}$. The $5 d$ orbital of the rare earth and the $3 d$ orbital of the $\mathrm{Cu}$ are located at energies $\Delta_{5 d}$ and $\Delta_{3 d}$, respectively. The second line in the Hamiltonian corresponds to the transfer part between the individual $4 f$ orbitals and the $\mathrm{Cu} 3 d$ orbital, with a transfer parameter $t_{3 d-4 f}$ and a transfer between the rare-earth $5 d$ orbital and the $\mathrm{Cu} 3 d$ orbital with a transfer parameter $t_{3 d-5 d}$. Here, we assume that the orbitals on the same site are eigenstates of the site Hamiltonian and therefore do not mix. The third line in the Hamiltonian corresponds to Hubbard electron repulsion terms $U_{f}, U_{5 d}$, and $U_{3 d}$, which are the repulsion parameters for the orbitals we have considered. The fourth line involves interorbital interactions on the rare-earth site with $U_{f f}$ for repulsion between electrons in different $4 f$ orbitals and $U_{f d}$ for repulsion between electrons in the $4 f$ and $5 d$ orbitals at the rare-earth site. The fifth line in the Hamiltonian corresponds to the intersite interaction between electrons on the rare-earth site and those in the $\mathrm{Cu} 3 d$ orbital. The parameters $V_{3 d-4 f}$ and $V_{3 d-5 d}$ are obtained using Ohno parametrization

$$
V_{i j}=14.397\left[\left(\frac{28.794}{U_{i}+U_{j}}\right)^{2}+r_{i j}^{2}\right]^{-1 / 2},
$$

with intersite distance of $3 \AA$, values are 4.04 and $3.46 \mathrm{eV}$, respectively. The last two lines in the Hamiltonian correspond to the exchange term involving orbitals on the rareearth site. The exchange term has both diagonal and offdiagonal contributions, the latter coming from two successive electron hops.

We set up the matrix for the above Hamiltonian in the valence bond (VB) basis. The VB basis being eigenstates of the total spin operator is ideal for this study since the total spin of the targeted state is known exactly. The VB method is discussed extensively in Ref. 23. The resulting Hamiltonian matrix is nonsymmetric and is diagonalized using Rettrup's algorithm. ${ }^{24}$ The low-energy states are determined for a range of values of the parameters in the Hamiltonian. These studies were carried out at various $4 f$ orbital fillings, corresponding to different lanthanide ions.

We have also carried out calculations that includes spinorbit interactions between the $4 f$ orbital angular momentum and the electron spin. The spin-orbit contribution is given by

$$
\hat{H}_{\mathrm{SO}}=\sum_{i} \xi\left(r_{i}\right) l(i) \cdot s(i),
$$

where $\xi\left(r_{i}\right)$ is given by

$$
\xi\left(r_{i}\right)=\frac{\hbar}{2 m^{2} c^{2} r} \frac{\partial V}{\partial r}
$$

with usual notations. Integrating over the radial part leads to the spin-orbit interaction parameter $\lambda$ and $\hat{H}_{\text {SO }}$ can be written in the second quantized form as 


$$
\begin{aligned}
\hat{H}_{\mathrm{SO}}= & \frac{\lambda}{2} \sum_{m}\left[\sqrt{l(l+1)-m(m+1)} \hat{a}_{m+1, \beta} \hat{a}_{m, \alpha}\right. \\
& +\sqrt{l(l+1)-m(m-1)} \hat{a}_{m-1, \alpha}^{\dagger} \hat{a}_{m, \beta} \\
& \left.+m\left(\hat{a}_{m, \alpha}^{\dagger} \hat{a}_{m, \alpha}-\hat{a}_{m, \beta}^{\dagger} \hat{a}_{m, \beta}\right)\right],
\end{aligned}
$$

where $l$ takes the value 3 , corresponding to the $f$ orbitals and $m$ varies from -3 to 3 . In the presence of spin-orbit interactions, the total spin of the system is not conserved. Thus, we cannot employ the VB method for their solution. Instead we employ a constant $M_{s}$ basis, typified by Slater determinants, to set up the Hamiltonian matrix. The Hamiltonian matrix in this case is symmetric and Davidson algorithm ${ }^{25}$ gives the low-lying states. Since the states are now labeled by the total angular momentum $J=L+S$, we compute the expectation value of $\hat{S}^{2}$ and use the nearest integer or halfinteger value $S$ such that the expectation value is nearest to $S(S+1)$. This allows identifying the approximate total spin of the states.

\section{RESULTS AND DISCUSSION}

The Hamiltonian in Eq. (2) has several parameters and exploring the phase space for all possible filling of the $4 f$ rare-earth orbital is very compute intensive. In order to meaningfully explore the parameter space, we have first identified the most sensitive parameters and fixed the remaining parameters at generally accepted values. We have considered three different models for the $\mathrm{Ln}-\mathrm{Cu}$ system. These correspond to (a) ignoring the $5 d$ levels on the rareearth ion, (b) including all the five $5 d$ orbitals on the rareearth ion, and (c) including only one $5 d$ orbital on the rareearth ion. In all these cases, we have found that the interorbital electron repulsion strength $U_{f f}$ in the $4 f$ orbital and the crystal-field splitting of the $4 f$ orbitals $\Delta_{f}$ are the parameters to which the spin of the ground state is most sensitive. The remaining parameters have been held fixed at the following values. The value of the exchange constants $J_{f f}$ and $J_{f d}$ are fixed at $0.1 U_{f}$ and $0.2 U_{f}$, respectively. ${ }^{26}$ The $U_{3 d}$ on copper is fixed at $5 \mathrm{eV},{ }^{26} t_{3 d-4 f}$ at $0.1 \mathrm{eV}$ and in all the models and in models (b) and (c) $t_{3 d-5 d}$ was fixed at 0.5 $\mathrm{eV}^{27}, U_{5 d}$ at $5 \mathrm{eV}$, and $U_{f d}$ at $4 \mathrm{eV}$. In what follows, we discuss our results, first for the half-filled $4 f$ shell and then for rare-earth ions with other $4 f$ occupancies, for various values of the parameters $U_{f f}$ and $\Delta_{f}$. In the last subsection we explore the effect of spin-orbit interactions on the "spin" of the ground state for these parameter values.

\section{A. $4 f^{7}$ system}

For the half-filled $\mathrm{Ln}$ ion in model (a) where the $5 d$ orbitals on the rare-earth site is ignored, we always obtain a low-spin ground state, for all values of $U_{f}$ and $\Delta_{f}$ that we scanned. Changing the parameters $t_{3 d-4 f}$ and $U_{3 d}$ within reasonable limits still leaves the system in a low-spin ground state. This is because the path way for delocalization of the electrons in the high-spin state is blocked [Fig. 2(a)], depriving the system of kinetic stabilization. Thus, this model does

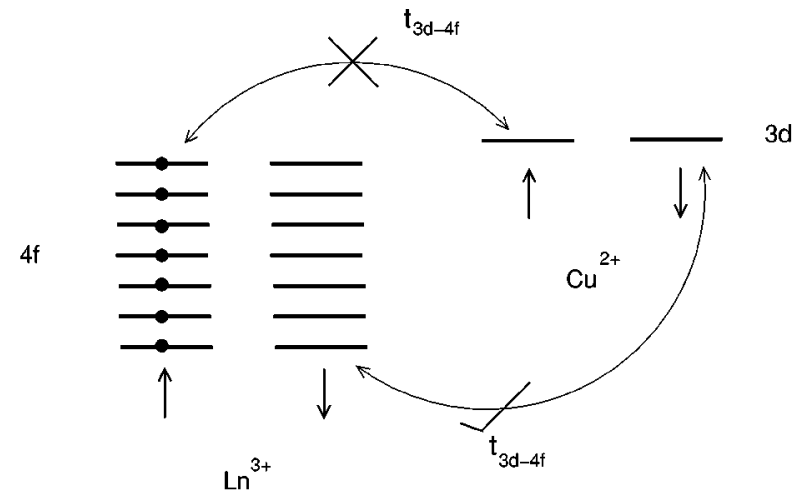

(a)

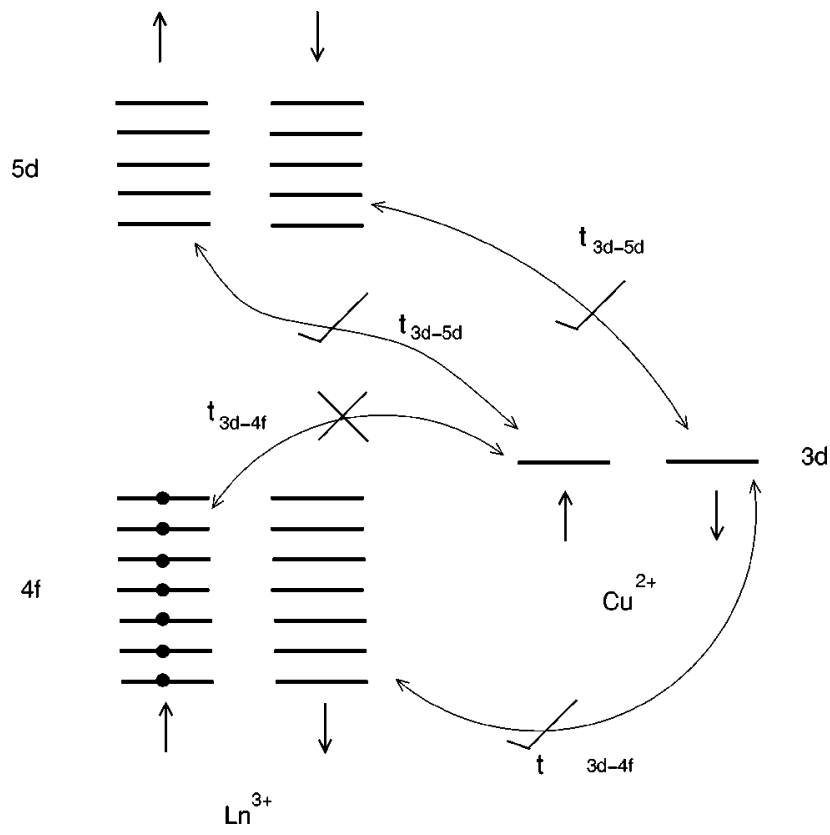

(b)

FIG. 2. A schematic diagram showing the various possible virtual states obtained by electron transfer from $3 d$ orbital of $\mathrm{Cu}$ (II) to orbitals of $\operatorname{Ln}$ (III), when $4 f$ is half-filled-(a) without $5 d$ levels, (b) with all the $5 d$ levels taken into consideration.

not produce a high-spin ground state for any value of $\Delta_{f}$ or $U_{f f}$, although experimentally most $4 f^{7}$-radical or $\mathrm{Cu}(\mathrm{II})$ systems are found in high-spin ground state.

In the presence of the empty $5 d$ orbitals [Fig. 2(b)], the Hunds rule stabilizes the state in which the $4 f$ and $5 d$ electrons have the same spin alignment. This in turn implies that the high-spin state in which the unpaired electron in the $\mathrm{Cu}$ $3 d$ orbital has the same orientation as the spins in the $4 f$ orbital has lower energy than the low-spin state. Our studies on model (b) is therefore expected to produce a high-spin ground state. However, what is quite unexpected is that we find a very interesting variation in the ground-state spin; the ground state is a high-spin state for small $\Delta_{f}(\leqslant 0.1 \mathrm{eV})$ and $1.5 \mathrm{eV} \leqslant U_{f f} \leqslant 4.5 \mathrm{eV}$ at $U_{f}=10 \mathrm{eV} .^{28}$ For other values of $U_{f f}$, the ground state is in a low-spin state. Thus, in the quantum phase diagram (Fig. 3), there is a reentrant low-spin 


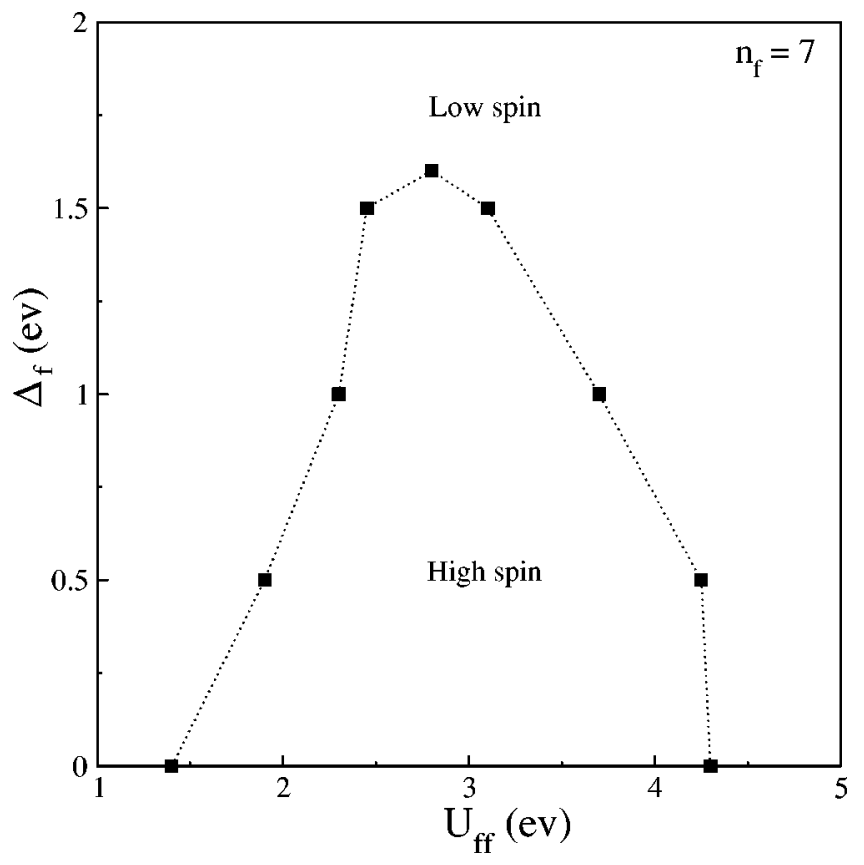

FIG. 3. Phase diagram in the space of parameters $U_{f f}$ (in eV) and $\Delta_{f}$ (in $\mathrm{eV}$ ), for the case of rare-earth $4 f$ levels being half filled. Other parameter values are as follows: $U_{f}=10 \mathrm{eV}, t_{3 d-4 f}$ $=0.1 \mathrm{eV}, t_{3 d-5 d}=0.5 \mathrm{eV}, U_{5 d}=5 \mathrm{eV}, U_{3 d}=5 \mathrm{eV}, U_{f d}=4 \mathrm{eV}, J_{f f}$ $=0.1 \mathrm{eV}$, and $J_{f d}=0.2 \mathrm{eV}$.

phase. For small values of $U_{f f}$ the virtual low-spin state in which the intermediate rare-earth ion configuration is $4 f^{8}$ has lower energy than the configuration $4 f^{7} 5 d^{1}$ and this would lead to a low-spin ground state of the complex. At intermediate values of $U_{f f}$, the virtual state $4 f^{7} 5 d^{1}$ has a

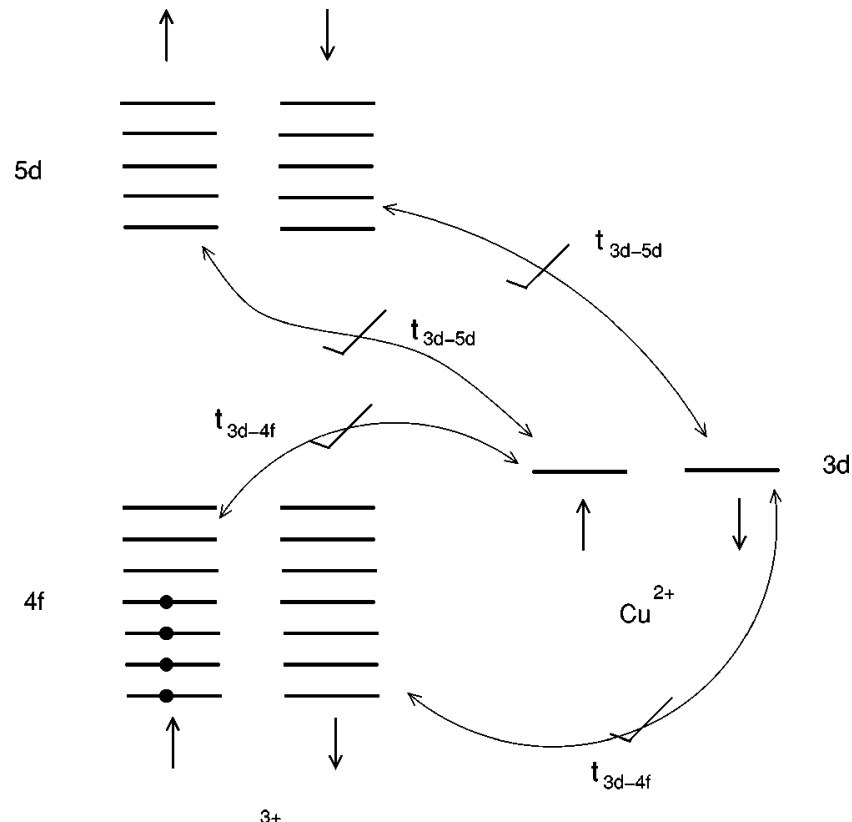

$\operatorname{Ln}^{3+}$

FIG. 4. A schematic diagram showing the various possible virtual states obtained by electron transfer from $3 d$ orbital of $\mathrm{Cu}$ (II) to orbitals of $\operatorname{Ln}(\mathrm{III})$, when $4 f$ is less than half filled.

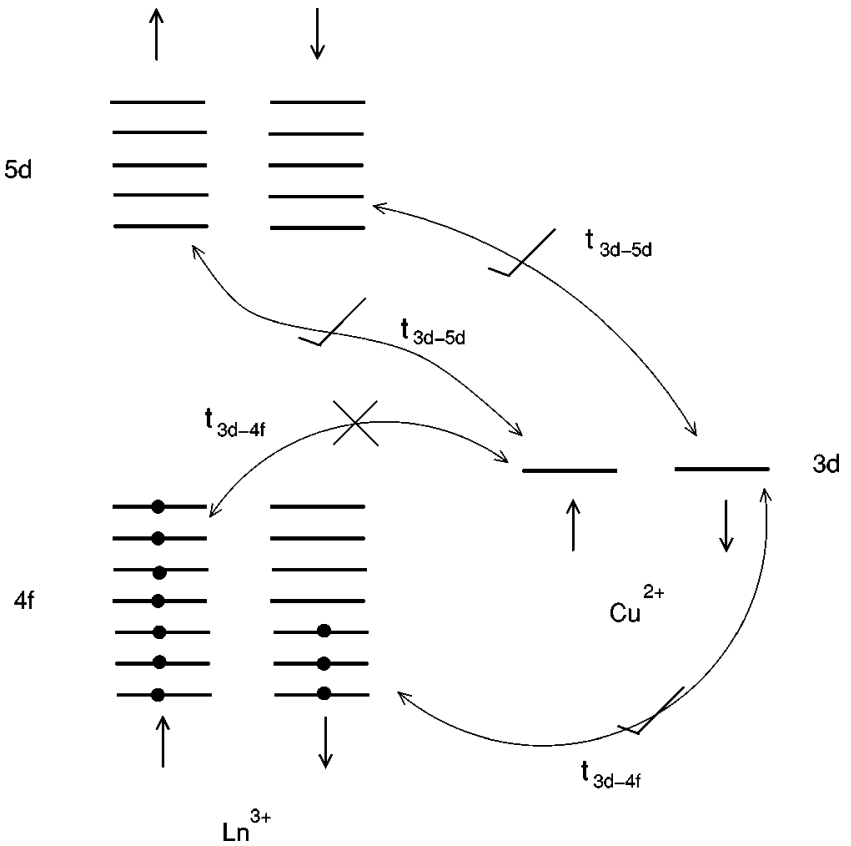

FIG. 5. A schematic diagram showing the various possible virtual states obtained by electron transfer from $3 d$ orbital of $\mathrm{Cu}$ (II) to orbitals of $\operatorname{Ln}(\mathrm{III})$, when $4 f$ is more than half filled.

lower energy than the $4 f^{8}$ virtual state and we should expect the ground state to be a high-spin state. For large values of $U_{f f}$, the ground-state configuration of the rare-earth ion is no longer $4 f^{7}$ but $4 f^{6} 5 d^{1}$. In this case, the hopping between the $5 d$ and $3 d$ orbitals favors antiparallel alignment of spins. The $3 d$ to $4 f$ hop prefers parallel alignment on energy consideration but there is only one channel for this process as against seven channels for antiparallel alignment. Therefore, the ground state switches back to low-spin state for high values of $U_{f f}$.

We also find that the high-spin state is quite stable and continues to be the ground state, even when the $t_{3 d-4 f}$ transfer integral is increased to unto twice its value of $0.1 \mathrm{eV}$ we have used in all our studies. Furthermore, we find that the $\Delta_{f}$ parameter which corresponds to the crystal-field splitting of the $4 f$ levels also plays a significant part; when $\Delta_{f}$ is small, the cross over from high-spin ground state to low-spin ground state occurs at a higher value of $U_{f f}$. Above a critical value of $\Delta_{f}$, the system is found only in the low-spin ground state (Fig. 3).

This picture is not changed in any significant way, in going to model (c) where only one $5 d$ orbital is used instead of the five $5 d$ orbitals on the rare earth considered in model (b). In view of this, in cases away from half-filled $4 f$ levels, we have considered only a single $5 d$ orbital [model (c)] for reasons of computational feasibility.

\section{B. Systems with non-half-filled $4 f$ shell}

We have studied systems with various $4 f$ orbital fillings using the Hamiltonian in Eq. (2) and the general result we find is that when $U_{f f}$ is small, systems with fewer than seven $4 f$ electrons have a high-spin ground state while those with 

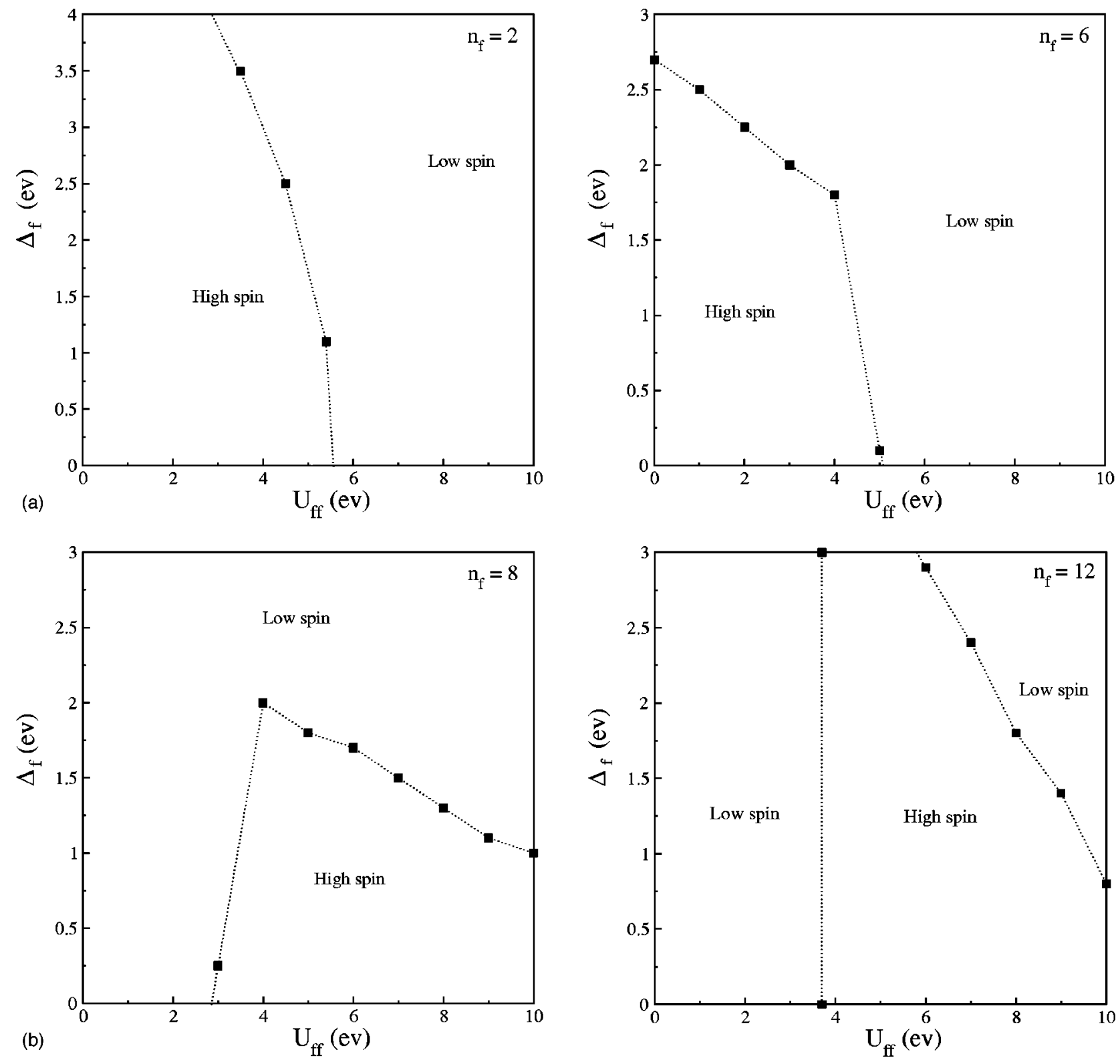

FIG. 6. Phase diagram in the space of parameters $U_{f f}$ (in $\mathrm{eV}$ ) and $\Delta_{f}$ (in eV), for the case of rare-earth $4 f$ levels being away from half filling. Other parameter values are same as in Fig. 3

more than seven $4 f$ electrons have a low-spin ground state (Fig. 6). This result follows even in model (a) where we have neglected the participation of the $5 d$ orbitals in delocalization. This result is quite simply understood from the direct exchange interaction term. In the less than half-filled case (Fig. 4), an electron hop from the $\mathrm{Cu} 3 d$ orbital to the rareearth $4 f$ orbital can result in a high-spin or a low-spin rareearth ion. If the unpaired electrons on the rare earth have the same alignment as the hopping electron, we obtain the highspin state, otherwise the resulting state of the rare-earth ion is a low-spin state. The direct exchange interaction within $4 f$ orbitals ensures that the virtual high-spin state of the ion has lower energy than the virtual low-spin state. Therefore, the ferromagnetic alignment of the spins on the $\mathrm{Ln}-\mathrm{Cu}$ system is favored over antiferromagnetic alignment in the less than half-filled case. In the more than half-filled case, electron- hop is only permitted when the hopping electron has an alignment opposite that of the rare-earth spin (Fig. 5), because of Pauli exclusion principle. Thus, in the more than half-filled case, we find a low-spin ground state. Indeed, this picture is not significantly changed when we go to model (c) where we have the copper $3 d$ electron having an additional delocalization pathway via the rare-earth $5 d$ orbital. What we also note is that the $t_{3 d-5 d}$ does not have a significant effect, unlike in the half-filled case.

We should expect the crystal-field splitting parameter $\Delta_{f}$ of the $4 f$ orbitals to play an important role in determining the ground state of the Ln-Cu system, since in case of large $\Delta_{f}$, the rare-earth ion ground state would not be a high-spin ground state. For a similar reason, we should also expect the interorbital repulsion $U_{f f}$ to determine the magnetic state of the dimer. In Fig. 6, we present some typical quantum phase 


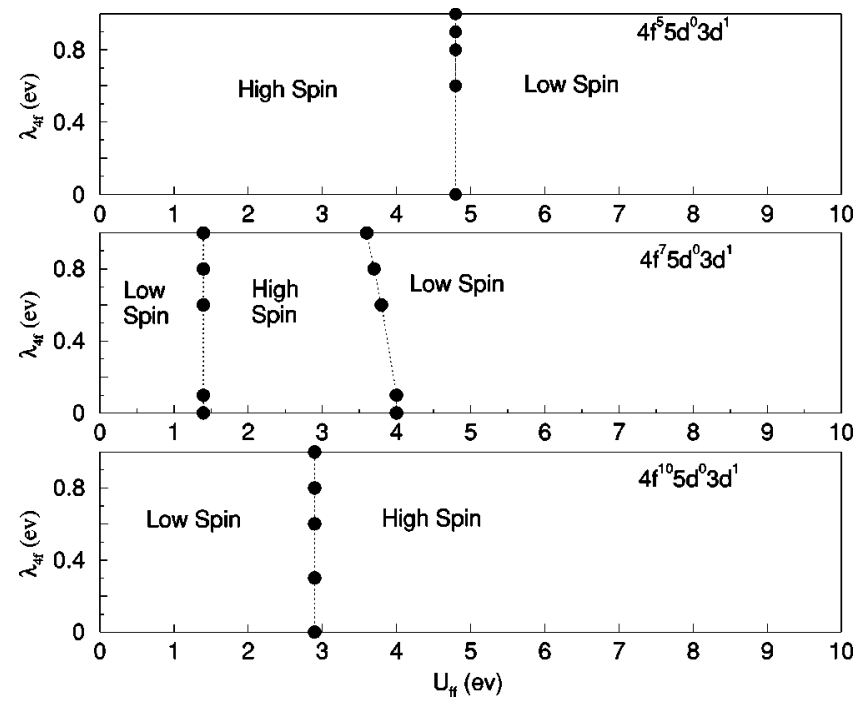

FIG. 7. Phase diagram in the space of parameters $U_{f f}$ (in eV) and $\lambda_{4 f}$ (in $\mathrm{eV}$ ), for the case of rare-earth $4 f$ levels at less than half filling, half-filling and more than half-filling. Other parameter values are same as in Fig. 3.

diagrams for the less than half-filled case as well as the more than half-filled case. In the less than half-filled case, for large $U_{f f}$, we obtain a low-spin ground state since the energy difference between high-spin and low-spin rare-earth virtual states obtained on electron transfer, is not significant. However, number of low-spin virtual states are more than the high-spin virtual states and we obtain a low-spin ground state for large $U_{f f}$. The experimental system with less than halffilled $4 f$ shell are all known to be in the low-spin (AFM) ground state. Thus, these systems all correspond to the large $U_{f f}$ limit. The critical value of $U_{f f}$ decreases with increase in $4 f$ occupancy from 1 to 7 .

In Fig. 6, we show some typical quantum phase diagrams for the more than half-filled case. Here again we note a quantum phase transition, from the low-spin ground state to the high-spin ground state, at large values of $U_{f f}$. This transition is also qualitatively understood in the following way. At large $U_{f f}$, the low-energy exchange path way is via the empty $5 d$ orbitals. The direct exchange interaction between the $5 d$ and $4 f$ orbitals on the rare-earth ensure that the virtual states corresponding to high-spin state has a lower energy than those corresponding to low-spin state. Thus, the dimer switches to a high-spin ground state with increasing $U_{f f}$. All the experimental systems known in this case correspond to high-spin ground state. Thus, we are in the large $U_{f f}$ limit in real systems even in systems in which the rareearth ion has more than half-filled $4 f$ shell. This is also intuitively expected since the $4 f$ shell is chemically well isolated in rare-earth compounds. The critical value of $U_{f f}$ in this case increases with increase in the $4 f$ occupancy beyond half filling.

\section{Role of spin-orbit interactions}

It is well known that the spin-orbit interactions in rareearth ions are important and often larger than the crystal-field

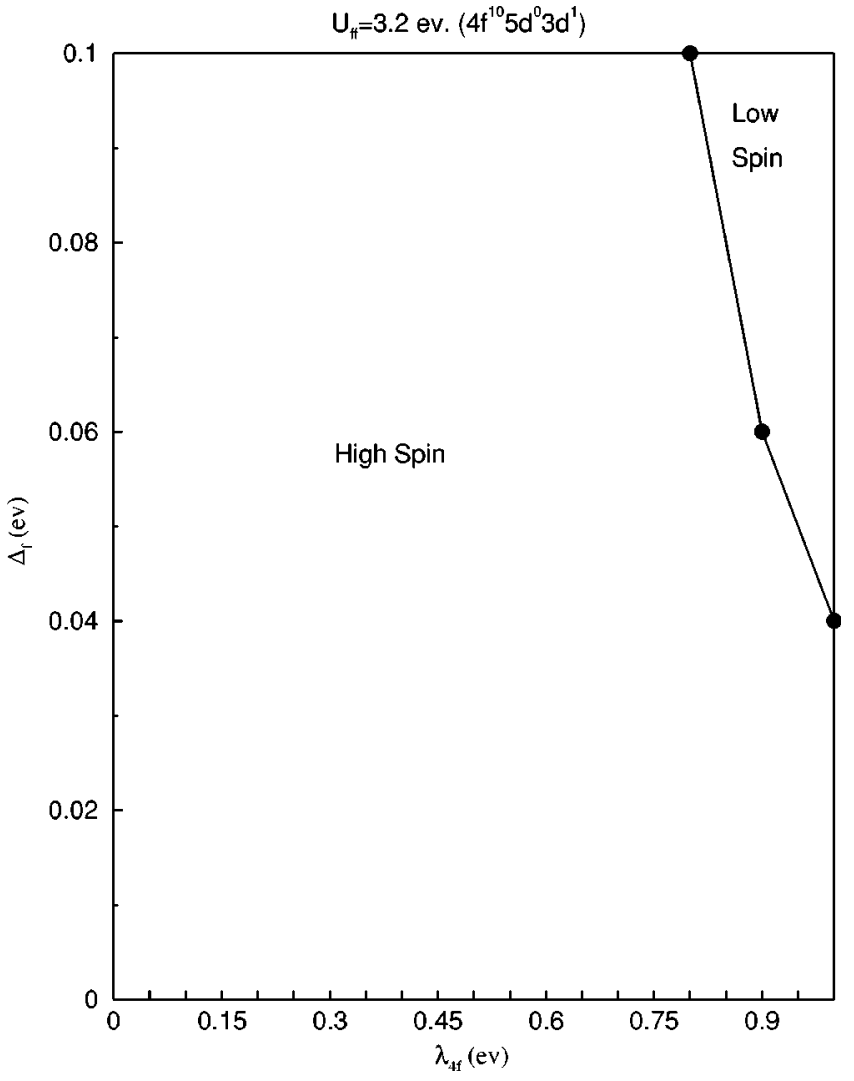

FIG. 8. Phase diagram in the space of parameters $\lambda_{4 f}$ (in $\mathrm{eV}$ ) and $\Delta_{f}$ (in eV) for the case of rare-earth $4 f$ levels being away from half-filling. Other parameter values are same as in Fig. 3.

effects. We have considered only the spin orbit interaction for electrons in the $4 f$ orbital of the rare earth and have ignored it in the $5 d$ orbital of the rare earth as well as in the $3 d$ orbital of the copper. Our method of calculation takes into account spin-orbit interactions at the same level as all other interactions in the Hamiltonian in Eq. (2), since we setup and solve the total Hamiltonian [Hamiltonians in Eq. (2) plus Eq. (4)]. Thus, our studies go beyond the usual $L-S$ or $j-j$ coupling schemes in which either the spin-orbit interaction is treated perturbatively within a given manifold of $L$ and $S$ or the electron repulsions are treated perturbatively over the individual spin-orbit coupled states. We have used values of $\lambda$ in the range 0 to $1.0 \mathrm{eV}$, for the $U_{f f}$ and $\Delta_{f}$ values. ${ }^{7}$ In the presence of spin-orbit interactions, the total spin is no longer a good quantum number. Thus we cannot strictly classify the eigenstates by their spins as low-spin or high-spin states. However, we find that the expectation value of $\hat{S}^{2}$ in the low-lying states, in all the cases we have studied, is close enough to allowed $S$ values in the absence of spinorbit interactions. This aids in labeling the states as "highspin" or "low-spin" states.

What we find is that the role of the spin-orbit interaction in determining the ground state magnetic moment of the $\mathrm{Ln}-\mathrm{Cu}$ dimers is negligible. In Fig. 7, we show the quantum phase diagram for three representative cases corresponding to less than half-filled, half-filled, and more than half-filled $4 f$ shells, for $\Delta_{f}=0$ as a function of $\lambda$ and $U_{f f}$, with all 


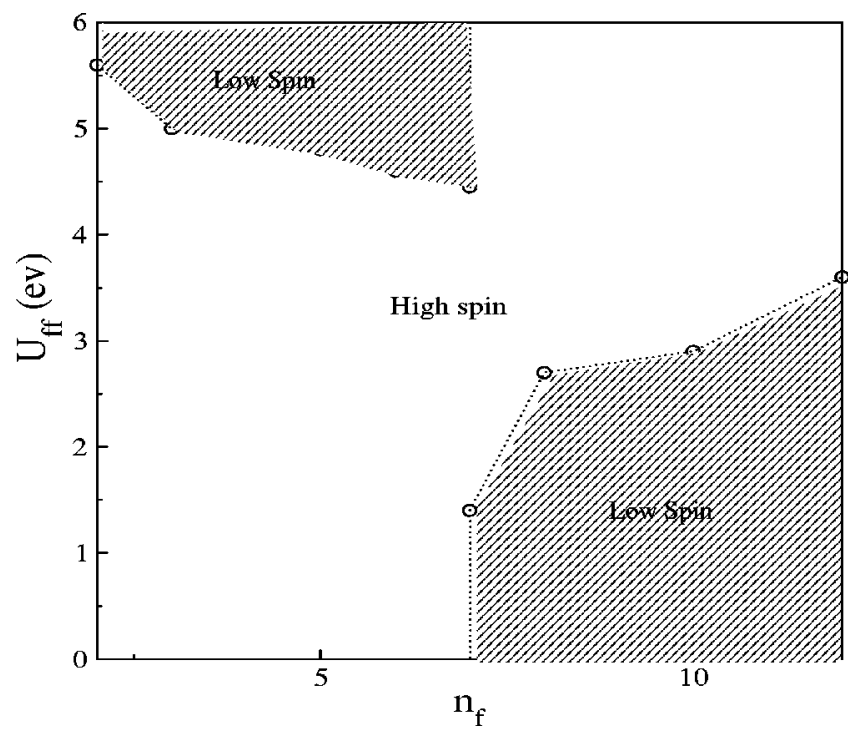

FIG. 9. A phase diagram for the $\mathrm{Ln}(\mathrm{III})-\mathrm{Cu}(\mathrm{II})$ system, between parameters $U_{f f}$ (in $\mathrm{eV}$ ) and $n_{f}, n_{f}$ being the $4 f$ level filling. $\Delta_{f}$ value was fixed at $0.1 \mathrm{eV}$. Other parameter values are same as in Fig. 3.

other parameters being the same as in Figs. 3 and 6. We find that the $U_{f f}$ value for which the magnetic transition occurs in the ground state is hardly affected by spin-orbit interactions. Only the reentrant low-spin state occurs for a slightly smaller value of $U_{f f}$ when spin-orbit interaction is sufficiently strong. We have also explored the role of spin-orbit interaction when the crystal field splitting of the $4 f$ orbitals $\Delta_{f}$ is varied near a critical value of $U_{f f}$. Here we assume that the splitting of the $f$-orbital manifold by the crystal field does not quench the orbital angular momentum of the resulting state. In Fig. 8 the phase diagram as a function of $\lambda$ for the case of the rare-earth electron configuration $4 f^{10}$ is shown. We have chosen this configuration as we found the strongest effect due to spin-orbit interactions in this case. We find in Fig. 7 that the high-spin state crosses over to the low-spin state at a smaller value of $\Delta_{f}$ when the spin-orbit interaction strength $\lambda$ is increased. Thus, we conclude from our studies that the magnetic state of the cluster is only weakly influenced by spin-orbit interactions.

\section{SUMMARY AND CONCLUSIONS}

In this paper, we have studied the magnetic ground state of the heterobimetallic organic molecular magnets, with a rare-earth ion and a copper ion. These systems have been modeled with a many-body Hamiltonian, which includes the direct exchange interaction and the repulsion between the orbitals on the same site. The systems are shown to exhibit a low-spin or high-spin ground state depending on the filling of electrons in the rare earth $4 f$ levels, and also critically on the inter-orbital $4 f$ electron repulsions $U_{f f}$ and the splitting of the degenerate $4 f$ orbitals in the presence of the crystal field $\Delta_{f}$. We have obtained a "phase diagram" in the space of these parameters. We find at half-filling a re-entrant lowspin state as $U_{f f}$ is increased for small values of $\Delta_{f}$. There is also a transition in the ground state spin at other fillings, again for small values of $\Delta_{f}$, when $U_{f f}$ is increased. The general phase diagram of the ground state spin is shown in Fig. 9 as a function of filling and $U_{f f}$. This picture is valid for $0 \leqslant \Delta_{f} \leqslant 1 \mathrm{eV}$ as for these values of $\Delta_{f}$ the low-spin and high-spin regime are seperated by a nearly constant $U_{f f}$. Furthermore, this picture is also not altered by the inclusion of spin-orbit interactions. To obtain the experimentally observed interaction, corresponding to low-spin ground state below half-filling and high-spin ground state above halffilling of the $4 f$ shell, we need to assume fairly large interorbital repulsions in the $4 f$ shell. Our studies show that in systems where the parameters are near the phase boundaries, small changes brought about by the modification of the ligands can lead to drastic changes in the magnetic properties of the complex.

\section{ACKNOWLEDGMENTS}

We thank Professor Diptiman Sen for many useful discussions. We thank the Council of Scientific and Industrial Research, India and Board of Research in Nuclear Sciences (BRNS) for financial support.
${ }^{1}$ W. Heitler and F. London, Z. Phys. 44, 455 (1927).

${ }^{2}$ For a review, see G. Blondin and J.J. Girerd, Chem. Rev. 90, 1359 (1990).

${ }^{3}$ O. Kahn, Molecular Magnetism (VCH, New York, 1993).

${ }^{4}$ A. Bencini, C. Benelli, A. Caneschi, A. Dei, and D. Gatteschi, Inorg. Chem. 25, 572 (1986).

${ }^{5}$ C. Benelli, A. Caneschi, D. Gatteschi, O. Guillou, and L. Pardi, Inorg. Chem. 29, 1750 (1990).

${ }^{6}$ O. Guillou, O. Kahn, R.L. Oushoorn, Boubekeur, and P. Batail, Inorg. Chim. Acta 198-200, 119 (1992).

${ }^{7}$ M.L. Kahn, J.P. Sutter, S. Golhen, P. Guionneau, L. Ouahab, O. Kahn, and D. Chasseau, J. Am. Chem. Soc. 122, 3413 (2000).

${ }^{8}$ J.P. Sutter, M.L. Kahn, and O. Kahn, Adv. Mater. 11, 863 (1999).

${ }^{9}$ J.P. Sutter and M.L. Kahn, Polyhedron 20, 1593 (2001).

${ }^{10}$ M. Andruh, I. Ramade, E. Codjvoi, O. Guillou, O. Kahn, and J.C.
Trombe, J. Am. Chem. Soc. 115, 1822 (1993).

${ }^{11}$ R. Pariser and R.G. Parr, J. Chem. Phys. 21, 446 (1953).

${ }^{12}$ K. Ohno, Theor. Chem. Acc. 2,219 (1964); G. Klopman, J. Am. Chem. Soc. 86, 4550 (1964); J.A. Pople, Trans. Faraday Soc. 49, 1375 (1953).

${ }^{13}$ J. B. Goodenough, Magnetism and Chemical Bond (Interscience, New York, 1963), p. 165.

${ }^{14}$ A.L. Tchougreeff, J. Chem. Phys. 96, 6026 (1992).

${ }^{15}$ P. Turek, K. Nozawa, D. Shiomi, K. Awaga, T. Inabe, Y. Maruyama, and M. Kinoshita, Chem. Phys. Lett. 180, 327 (1991).

${ }^{16}$ R. Hotzelmann, K. Wieghardt, U. Flörke, H.J. Haupt, D.C. Weatherburn, J. Bonvoisin, G. Blondin, and J.J. Girerd, J. Am. Chem. Soc. 114, 1681 (1992).

${ }^{17}$ Bhabadyuti Sinha and S. Ramasesha, Phys. Rev. B 48, 16410 
(1993).

${ }^{18}$ H. Ogasawara, A. Kotani, P.L. Fevre, D. Chandesris, and H. Magnan, Phys. Rev. B 62, 7970 (2000).

${ }^{19}$ C.J. Ballhausen, Introduction to Ligand Field Theory (McGrawHill, New York, 1962).

${ }^{20}$ C. Lescop, D. Luneau, E. Belorizky, P. Fries, M. Guillot, and P. Rey, Inorg. Chem. 38, 5472 (1999).

${ }^{21}$ J.P. Costes, F. Dahan, and A. Dupuis, Inorg. Chem. 39, 169 (2000)

${ }^{22}$ A. Caneschi, A. Dei, D. Gatteschi, L. Sorace, and K. Vostrikova, Angew. Chem. Int. Ed. Engl. 39, 246 (2000).
${ }^{23}$ For a review, see Z. G. Soos and S. Ramasesha in Valence Bond Theory and Chemical Structure, edited by D. J. Klein and N. Trinajstić (Elsevier, Amsterdam, 1990).

${ }^{24}$ S. Rettrup, J. Comput. Phys. 45, 100 (1982).

${ }^{25}$ E.R. Davidson, J. Comput. Phys. 17, 87 (1975).

${ }^{26}$ J.E. Hirsch, Phys. Rev. B 56, 11022 (1997).

${ }^{27} \mathrm{O}$. Kahn and O. Guillou in Research Frontiers in Magnetochemistry, edited by C.J.O' Connor (World Scientific, Singapore, 1993).

${ }^{28}$ W. Nolting, W. Borgiel, and G. Borstel, Phys. Rev. B 37, 7663 (1988). 